\title{
Fundamental Solutions to Time-Fractional Advection Diffusion Equation in a Case of Two Space Variables
}

\author{
Y. Z. Povstenko \\ Institute of Mathematics and Computer Science, Jan Dlugosz University in Częstochowa, Waszyngtona 4/8, 42-200 Częstochowa, \\ Poland \\ Correspondence should be addressed to Y. Z. Povstenko; j.povstenko@ajd.czest.pl
}

Received 28 December 2013; Accepted 6 February 2014; Published 11 March 2014

Academic Editor: J. A. Tenreiro Machado

Copyright (C) 2014 Y. Z. Povstenko. This is an open access article distributed under the Creative Commons Attribution License, which permits unrestricted use, distribution, and reproduction in any medium, provided the original work is properly cited.

\begin{abstract}
The fundamental solutions to time-fractional advection diffusion equation in a plane and a half-plane are obtained using the Laplace integral transform with respect to time $t$ and the Fourier transforms with respect to the space coordinates $x$ and $y$. The Cauchy, source, and Dirichlet problems are investigated. The solutions are expressed in terms of integrals of Bessel functions combined with Mittag-Leffler functions. Numerical results are illustrated graphically.
\end{abstract}

\section{Introduction}

The classical advection diffusion equation

$$
\frac{\partial c}{\partial t}=a \Delta c-\mathbf{v} \cdot \nabla c
$$

where $a$ is the diffusivity coefficient, $\mathbf{v}$ is the velocity vector, has several physical interpretations in terms of Brownian motion, diffusion or heat transport with external force or with additional velocity field, diffusion of charge in the electrical field on comb structure, transport processes in porous media, groundwater hydrology, and so forth [1-7].

In the case of one spatial coordinate $x$, (1) has the following form:

$$
\frac{\partial c}{\partial t}=a \frac{\partial^{2} c}{\partial x^{2}}-v \frac{\partial c}{\partial x} .
$$

Investigation of different physical phenomena in media with complex internal structure has led to considering differential equations with derivatives of fractional order. The space-fractional [8-19], time-fractional [20-31], and spacetime-fractional [32-39] generalizations of the advection diffusion equation were studied by many authors. In the majority of the abovementioned papers, the fractional generalizations of one-dimensional equation (2) were considered. In the papers dealing with space-fractional or space-time-fractional equations, one term with space derivative was substituted by the corresponding term with the fractional derivative $[8,9,11-19,33,39]$ or both terms with space derivatives had fractional order [32, 35-38]. Several numerical schemes were proposed: the implicit difference method based on the shifted Grünwald-Letnikov approximation [14, 37], the explicit difference scheme [37], transformation of fractional differential equation into a system of ordinary differential equations and using the method of lines [15], the random walk algorithms $[16,17]$, the spectral regularization method [28], the Crank-Nicholson difference scheme [29], Adomian's decomposition [26], a spatial and temporal discretization $[30,39]$, the fractional variational iteration method [31], and the homotopy perturbation method [27, 38].

In $[24,25]$, the analytical solution to one-dimensional time-fractional advection diffusion equation was obtained in terms of integrals of the $H$-function.

In this paper, we study the fundamental solutions to timefractional advection diffusion equation

$$
\frac{\partial^{\alpha} c}{\partial t^{\alpha}}=a \Delta c-\mathbf{v} \cdot \nabla c
$$

in a plane and a half-plane. The Laplace transform with respect to time and the Fourier transform with respect to the space coordinates are used. The Cauchy and the source problems in a plane and the Dirichlet problem for a half-plane are solved. The analytical solutions are expressed in terms of 
integrals of the Mittag-Leffler functions. Numerical results are illustrated graphically.

In (3) we use the Caputo fractional derivative [40-42]:

$$
\frac{\mathrm{d}^{\alpha} c(t)}{\mathrm{d} t^{\alpha}}=\left\{\begin{array}{lc}
\frac{1}{\Gamma(n-\alpha)} \int_{0}^{t}(t-\tau)^{n-\alpha-1} \frac{\mathrm{d}^{n} c(\tau)}{\mathrm{d} \tau^{n}} \mathrm{~d} \tau, \\
\frac{\mathrm{d}^{n} c(t)}{\mathrm{d} t^{n}}, \quad n-1<\alpha<n,
\end{array}\right.
$$

where $\Gamma(\alpha)$ is the gamma function. For its Laplace transform rule, the Caputo fractional derivative requires the knowledge of the initial values of the function $c(t)$ and its integer derivatives of order $k=1,2, \ldots, n-1$ :

$$
\begin{array}{r}
\mathscr{L}\left\{\frac{\mathrm{d}^{\alpha} c(t)}{\mathrm{d} t^{\alpha}}\right\}=s^{\alpha} \mathscr{L}\{c(t)\}-\sum_{k=0}^{n-1} c^{(k)}\left(0^{+}\right) s^{\alpha-1-k}, \\
n-1<\alpha<n,
\end{array}
$$

where $s$ is the transform variable.

\section{The Fundamental Solution to the Cauchy Problem}

Consider the time-fractional advection diffusion equation

$$
\begin{array}{r}
\frac{\partial^{\alpha} c}{\partial t^{\alpha}}=a\left(\frac{\partial^{2} c}{\partial x^{2}}+\frac{\partial^{2} c}{\partial y^{2}}\right)-v \frac{\partial c}{\partial x}-v \frac{\partial c}{\partial y} \\
-\infty<x<\infty,-\infty<y<\infty \\
0<t<\infty, 0<\alpha \leq 1,
\end{array}
$$

under initial condition

$$
t=0: \quad c=p_{0} \delta(x) \delta(y) .
$$

In (7) we have introduced the constant multiplier $p_{0}$ to obtain the nondimensional quantity $\bar{c}$ (see (23)) displayed in Figures.

The zero conditions at infinity are also imposed:

$$
\lim _{x \rightarrow \pm \infty} c(x, y, t)=0, \quad \lim _{y \rightarrow \pm \infty} c(x, y, t)=0 .
$$

Introducing the new sought function

$$
c(x, y, t)=\exp \left[\frac{v(x+y)}{2 a}\right] u(x, y, t)
$$

and taking into account that for the Dirac delta function, $f(x) \delta(x)=f(0) \delta(x)$, the initial-value problem (6)-(8) is reduced to the following ones:

$$
\begin{gathered}
\frac{\partial^{\alpha} u}{\partial t^{\alpha}}=a\left(\frac{\partial^{2} u}{\partial x^{2}}+\frac{\partial^{2} u}{\partial y^{2}}\right)-\frac{v^{2}}{2 a} u, \\
t=0: \quad u=p_{0} \delta(x) \delta(y), \\
\lim _{x \rightarrow \pm \infty} u(x, y, t)=0, \quad \lim _{y \rightarrow \pm \infty} u(x, y, t)=0 .
\end{gathered}
$$

Next, we use the Laplace transform with respect to time $t$ (designated by the asterisk) and the double exponential Fourier transform with respect to the space coordinates $x$ and $y$ (marked by the tilde). In the transform domain, we get

$$
\widetilde{\widetilde{u}}^{*}=\frac{p_{0}}{2 \pi} \frac{s^{\alpha-1}}{s^{\alpha}+a\left(\xi^{2}+\eta^{2}\right)+v^{2} / 2 a} .
$$

Here, $s$ is the Laplace transform variable and $\xi$ and $\eta$ are the Fourier transform variables.

Inversion of the integral transforms gives

$$
\begin{aligned}
& u(x, y, t) \\
& =\frac{p_{0}}{4 \pi^{2}} \int_{-\infty}^{\infty} \int_{-\infty}^{\infty} E_{\alpha}\left\{-\left[a\left(\xi^{2}+\eta^{2}\right)+\frac{v^{2}}{2 a}\right] t^{\alpha}\right\} \\
& \times \cos (x \xi) \cos (y \eta) \mathrm{d} \xi \mathrm{d} \eta
\end{aligned}
$$

where the formula [40-42]

$$
\mathscr{L}^{-1}\left\{\frac{s^{\alpha-1}}{s^{\alpha}+b}\right\}=E_{\alpha}\left(-b t^{\alpha}\right)
$$

has been used with $E_{\alpha}(z)$ being the Mittag-Leffler function in one parameter $\alpha$ :

$$
E_{\alpha}(z)=\sum_{k=0}^{\infty} \frac{z^{k}}{\Gamma(\alpha k+1)}, \quad \alpha>0, z \in C .
$$

Solution (14) is not convenient for numerical calculations. To obtain the solution amenable to numerical treatment, we introduce the polar coordinates in the $(\xi, \eta)$-plane:

$$
\xi=\rho \cos \theta, \quad \eta=\rho \sin \theta
$$

Hence,

$$
\begin{aligned}
& u(x, y, t) \\
& =\frac{p_{0}}{4 \pi^{2}} \int_{0}^{\infty} \int_{0}^{2 \pi} E_{\alpha}\left[-\left(a \rho^{2}+\frac{v^{2}}{2 a}\right) t^{\alpha}\right] \\
& \quad \times \cos (x \rho \cos \theta) \cos (y \rho \sin \theta) \rho \mathrm{d} \rho \mathrm{d} \theta .
\end{aligned}
$$

Due to periodic properties of the integrand

$$
\begin{aligned}
& \int_{0}^{2 \pi} \cos (x \rho \cos \theta) \cos (y \rho \sin \theta) \mathrm{d} \theta \\
& \quad=4 \int_{0}^{\pi / 2} \cos (x \rho \cos \theta) \cos (y \rho \sin \theta) \mathrm{d} \theta .
\end{aligned}
$$

Changing variable $w=\sin \theta$ and taking into account the following integral [43]:

$$
\begin{gathered}
\int_{0}^{1} \frac{\cos \left(p \sqrt{1-x^{2}}\right)}{\sqrt{1-x^{2}}} \cos (q x) d x \\
=\frac{\pi}{2} J_{0}\left(\sqrt{p^{2}+q^{2}}\right),
\end{gathered}
$$




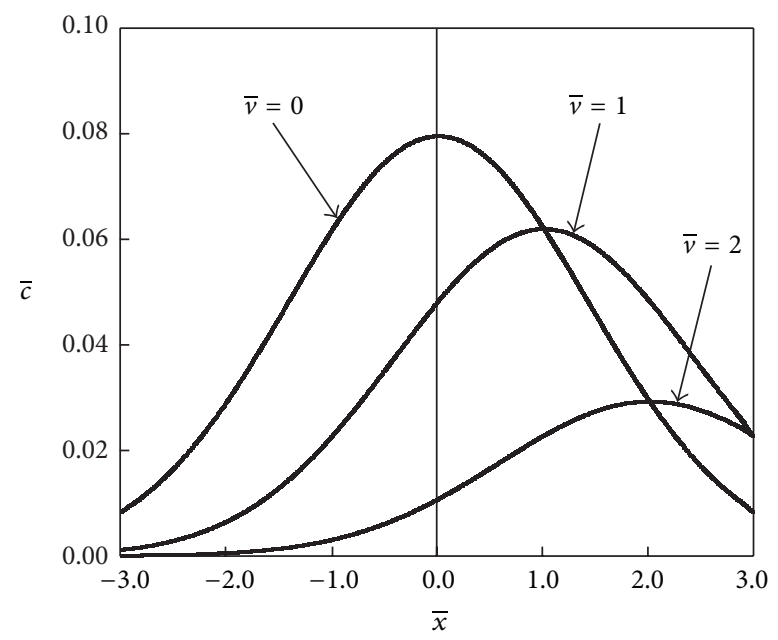

Figure 1: Dependence of the fundamental solution to the Cauchy problem on distance (the classical advection diffusion equation, $\alpha=$ $1)$.

where $J_{n}(z)$ is the Bessel function of the order $n$, we arrive at

$$
\begin{array}{r}
u(x, y, t)=\frac{p_{0}}{2 \pi} \int_{0}^{\infty} E_{\alpha}\left[-\left(a \rho^{2}+\frac{v^{2}}{2 a}\right) t^{\alpha}\right] \\
\times J_{0}\left(\sqrt{x^{2}+y^{2}} \rho\right) \rho \mathrm{d} \rho
\end{array}
$$

and, returning to the quantity $c(x, y, t)$ according to (9), we get

$$
\begin{aligned}
c(x, y, t)= & \frac{p_{0}}{2 \pi} \exp \left[\frac{v(x+y)}{2 a}\right] \\
& \times \int_{0}^{\infty} E_{\alpha}\left[-\left(a \rho^{2}+\frac{v^{2}}{2 a}\right) t^{\alpha}\right] \\
& \times J_{0}\left(\sqrt{x^{2}+y^{2}} \rho\right) \rho \mathrm{d} \rho .
\end{aligned}
$$

The particular case of solution (22) corresponding to the time-fractional diffusion equation $(v=0)$ was considered in $[44,45]$.

The results of numerical computations for $y=0$ are presented in Figure 1 for $\alpha=1$ and in Figure 2 for $\alpha=0.5$.

The following nondimensional quantities:

$$
\bar{c}=\frac{a t^{\alpha}}{p_{0}} c, \quad \bar{v}=\frac{t^{\alpha / 2}}{\sqrt{a}} v
$$

and the nondimensional coordinates (the similarity variables)

$$
\bar{x}=\frac{x}{\sqrt{a} t^{\alpha / 2}}, \quad \bar{y}=\frac{y}{\sqrt{a} t^{\alpha / 2}}
$$

have been introduced.

To calculate the Mittag-Leffler function $E_{\alpha}(-x)$ in solution (22), we applied the algorithm suggested in [46].

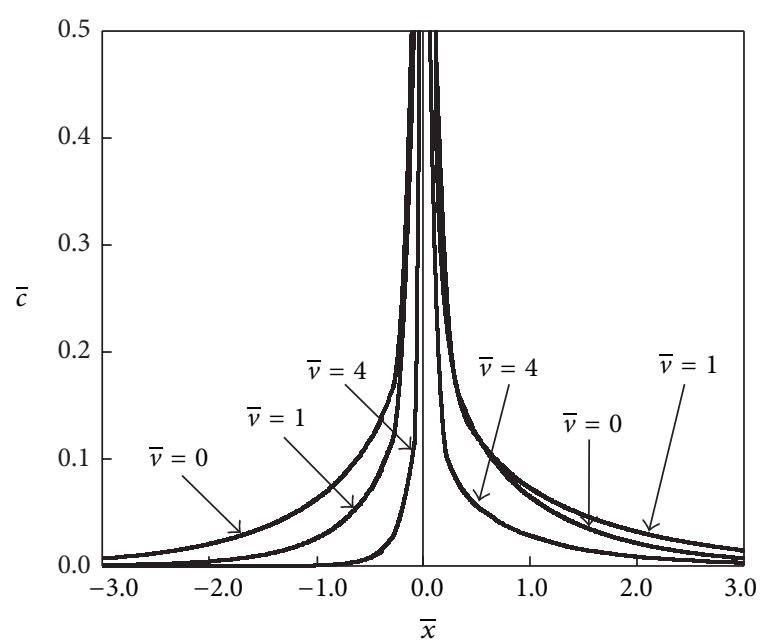

FIgUre 2: Dependence of the fundamental solution to the Cauchy problem on distance (the time-fractional advection diffusion equation, $\alpha=0.5$ ).

\section{The Fundamental Solution to the Source Problem}

Consider the time-fractional advection diffusion equation with the source term

$$
\begin{gathered}
\frac{\partial^{\alpha} c}{\partial t^{\alpha}}=a\left(\frac{\partial^{2} c}{\partial x^{2}}+\frac{\partial^{2} c}{\partial y^{2}}\right)-v \frac{\partial c}{\partial x}-v \frac{\partial c}{\partial y} \\
+q_{0} \delta(x) \delta(y) \delta(t), \\
-\infty<x<\infty, \\
-\infty<y<\infty, \\
0<t<\infty, 0<\alpha \leq 1,
\end{gathered}
$$

under zero initial condition,

$$
t=0: \quad c=0
$$

and conditions (8) at infinity.

The integral transform technique leads to

$$
\begin{gathered}
\tilde{\widetilde{u}}^{*}=\frac{q_{0}}{2 \pi} \frac{1}{s^{\alpha}+a\left(\xi^{2}+\eta^{2}\right)+v^{2} / 2 a}, \\
c(x, y, t)=\frac{q_{0} t^{\alpha-1}}{2 \pi} \exp \left[\frac{v(x+y)}{2 a}\right] \\
\quad \times \int_{0}^{\infty} E_{\alpha, \alpha}\left[-\left(a \rho^{2}+\frac{v^{2}}{2 a}\right) t^{\alpha}\right] \\
\quad \times J_{0}\left(\sqrt{x^{2}+y^{2}} \rho\right) \rho \mathrm{d} \rho .
\end{gathered}
$$

Here, $E_{\alpha, \beta}(z)$ is the generalized Mittag-Leffler function in two parameters $\alpha$ and $\beta$ :

$$
E_{\alpha, \beta}(z)=\sum_{k=0}^{\infty} \frac{z^{k}}{\Gamma(\alpha k+\beta)}, \quad \alpha>0, \beta>0, z \in C,
$$


and the formula [40-42]

$$
\mathscr{L}^{-1}\left\{\frac{s^{\alpha-\beta}}{s^{\alpha}+b}\right\}=t^{\beta-1} E_{\alpha, \beta}\left(-b t^{\alpha}\right)
$$

for the inverse Laplace transform has been used.

The particular case of solution (28) corresponding to the time-fractional diffusion equation with $v=0$ was considered in $[45,47]$. Solutions (22) and (28) coincide for $\alpha=1$.

The results of numerical computations for $y=0$ are presented in Figure 3 for $\alpha=0.5$ with

$$
\bar{c}=\frac{a t}{q_{0}} c
$$

\section{The Fundamental Solution to the Dirichlet Problem}

In this case the time-fractional advection diffusion equation,

$$
\begin{array}{r}
\frac{\partial^{\alpha} c}{\partial t^{\alpha}}=a\left(\frac{\partial^{2} c}{\partial x^{2}}+\frac{\partial^{2} c}{\partial y^{2}}\right)-v \frac{\partial c}{\partial x}-v \frac{\partial c}{\partial y} \\
0<x<\infty,-\infty<y<\infty \\
0<t<\infty, 0<\alpha \leq 1,
\end{array}
$$

is considered under zero initial condition

$$
t=0: \quad c=0
$$

and the Dirichlet boundary condition

$$
x=0: \quad c=g_{0} \delta(y) \delta(t) .
$$

The zero conditions at infinity are imposed as follows:

$$
\lim _{x \rightarrow \infty} c(x, y, t)=0, \quad \lim _{y \rightarrow \pm \infty} c(x, y, t)=0 .
$$

As above, the new sought function $u$ is introduced (see (9)), and, for (10) in the half-plane $x>0$, the Laplace transform with respect to time $t$, the exponential Fourier transform with respect to the spatial coordinate $y$, and the sin-Fourier transform with respect to the spatial coordinate $x$ are used. In the transform domain, we get

$$
\widetilde{\widetilde{u}}^{*}=\frac{a g_{0} \xi}{\sqrt{2 \pi}} \frac{1}{s^{\alpha}+a\left(\xi^{2}+\eta^{2}\right)+v^{2} / 2 a}
$$

and, after inversion of the integral transforms,

$$
\begin{aligned}
& u(x, y, t) \\
& =\frac{a g_{0} t^{\alpha-1}}{\pi^{2}} \int_{-\infty}^{\infty} \int_{0}^{\infty} E_{\alpha, \alpha}\left\{-\left[a\left(\xi^{2}+\eta^{2}\right)+\frac{v^{2}}{2 a}\right] t^{\alpha}\right\} \\
& \times \sin (x \xi) \cos (y \eta) \xi \mathrm{d} \xi \mathrm{d} \eta .
\end{aligned}
$$

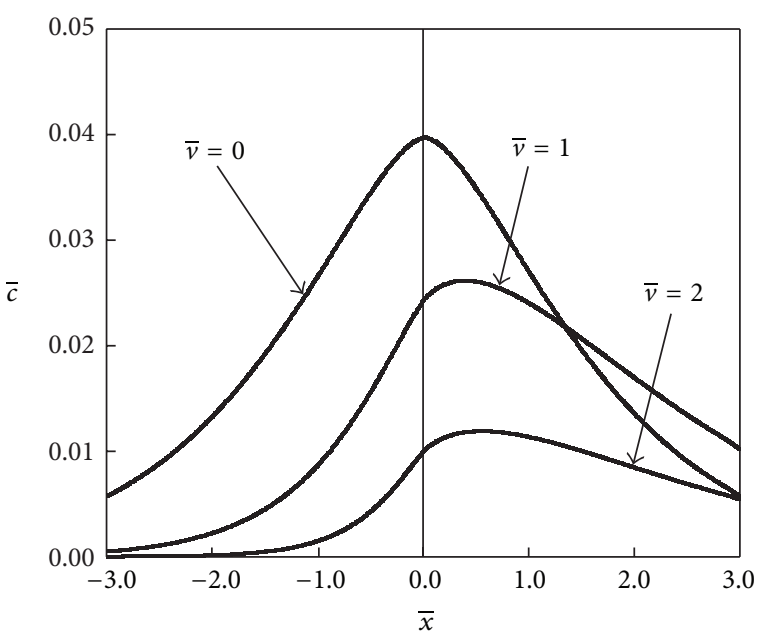

FIGURE 3: Dependence of the fundamental solution to the source problem on distance (the time-fractional advection diffusion equation, $\alpha=0.5$ ).

Introducing the polar coordinates in the $(\xi, \eta)$-plane gives

$$
\begin{aligned}
& u(x, y, t) \\
& =\frac{a g_{0} t^{\alpha-1}}{\pi^{2}} \int_{0}^{\infty} \int_{0}^{\pi} E_{\alpha}\left[-\left(a \rho^{2}+\frac{v^{2}}{2 a}\right) t^{\alpha}\right] \\
& \quad \times \sin (x \rho \cos \theta) \\
& \quad \times \cos (y \rho \sin \theta) \rho^{2} \cos \theta \mathrm{d} \rho \mathrm{d} \theta .
\end{aligned}
$$

Changing variables $w=\sin \theta$ and taking into account the following integral [43]:

$$
\begin{aligned}
& \int_{0}^{1} \sin \left(p \sqrt{1-x^{2}}\right) \cos (q x) \mathrm{d} x \\
& \quad=\frac{\pi}{2} \frac{p}{\sqrt{p^{2}+q^{2}}} J_{1}\left(\sqrt{p^{2}+q^{2}}\right),
\end{aligned}
$$

we obtain

$$
\begin{aligned}
u(x, y, t)= & \frac{a g_{0} t^{\alpha-1} x}{\pi \sqrt{x^{2}+y^{2}}} \int_{0}^{\infty} E_{\alpha, \alpha}\left[-\left(a \rho^{2}+\frac{v^{2}}{2 a}\right) t^{\alpha}\right] \\
& \times J_{1}\left(\sqrt{x^{2}+y^{2}} \rho\right) \rho^{2} \mathrm{~d} \rho \\
c(x, y, t)= & \frac{a g_{0} t^{\alpha-1} x}{\pi \sqrt{x^{2}+y^{2}}} \exp \left[\frac{v(x+y)}{2 a}\right] \\
& \times \int_{0}^{\infty} E_{\alpha, \alpha}\left[-\left(a \rho^{2}+\frac{v^{2}}{2 a}\right) t^{\alpha}\right] \\
& \quad \times J_{1}\left(\sqrt{x^{2}+y^{2} \rho}\right) \rho^{2} \mathrm{~d} \rho .
\end{aligned}
$$

The particular case of solution (41) corresponding to the time-fractional diffusion equation $(v=0)$ was considered in [48]. 


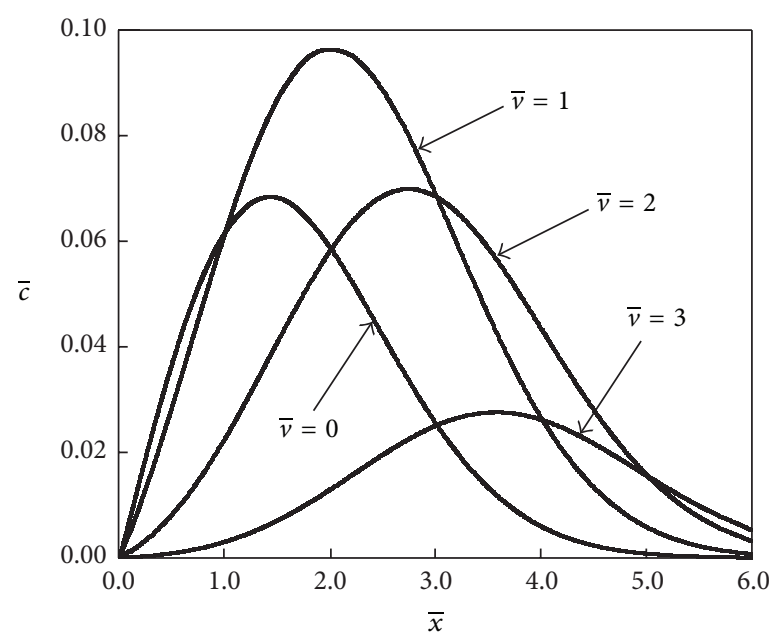

Figure 4: Dependence of the fundamental solution to the Dirichlet problem on distance (the classical advection diffusion equation, $\alpha=$ $1)$.

The results of numerical computations according to solution (41) for $y=0$ are presented in Figure 4 for $\alpha=1$ and in Figure 5 for $\alpha=0.5$ with

$$
\bar{c}=\frac{\sqrt{a} t^{1+\alpha / 2}}{g_{0}} c .
$$

Other nondimensional quantities are the same as in (23) and (24).

\section{Conclusions}

We have considered the time-fractional advection diffusion equation in a plane and in a half-plane. The fundamental solutions to the Cauchy problem and to the source problem in a plane have been obtained as well as to the Dirichlet problem in a half-plane. It should be emphasized that the fundamental solution to the Cauchy problem in the case $0<\alpha<1$ has the logarithmic singularity at the origin:

$$
\begin{aligned}
c(x, y, t) \sim & -\frac{p_{0}}{2 \pi \Gamma(1-\alpha) a t^{\alpha}} \exp \left[\frac{v(x+y)}{2 a}\right] \\
& \times \ln \left(\sqrt{1+\frac{v^{2} t^{\alpha}}{2 a}} \frac{\sqrt{x^{2}+y^{2}}}{\sqrt{a} t^{\alpha / 2}}\right) .
\end{aligned}
$$

This result is similar to the case of the time-fractional diffusion equation when $v=0$ (see $[44,49])$. Such a singularity disappears only for the classical advection diffusion equation $(\alpha=1)$. Due to singularity of the solution at the origin, in the case of $0<\alpha<1$, drift caused by the quantity $v$ is less noticeable than in the case of $\alpha=1$ (compare Figures 1 and 2).

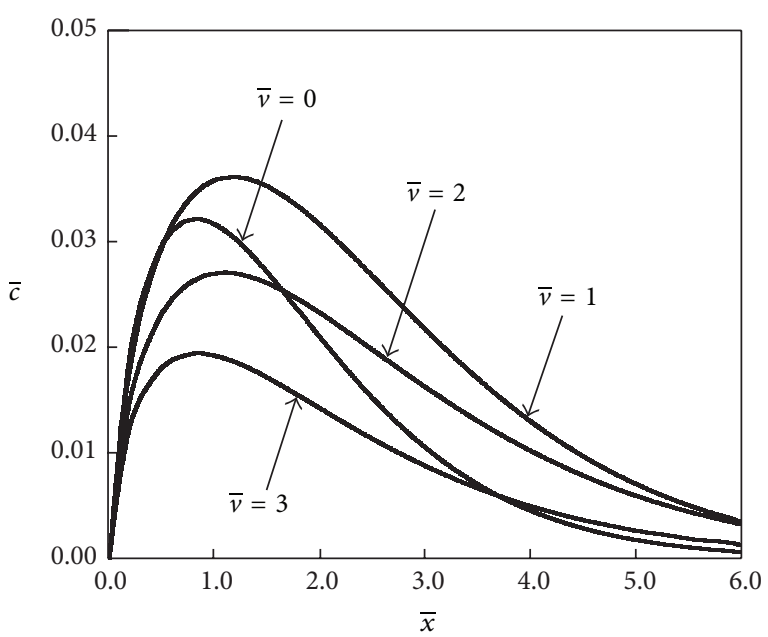

FIGURE 5: Dependence of the fundamental solution to the Dirichlet problem on distance (the time-fractional advection diffusion equation, $\alpha=0.5$ ).

\section{Conflict of Interests}

The author declares that there is no conflict of interests regarding the publication of this paper.

\section{References}

[1] W. Feller, An Introduction to Probability Theory and Its Applications, John Wiley \& Sons, New York, NY, USA, 2nd edition, 1971.

[2] A. E. Scheidegger, The Physics of Flow through Porous Media, University of Toronto Press, Toronto, Canada, 3rd edition, 1974.

[3] N. G. van Kampen, Stochastic Processes in Physics and Chemistry, North-Holland, Amsterdam, The Netherlands, 1981.

[4] H. Risken, The Fokker-Planck Equation, Springer, Berlin, Germany, 1989.

[5] M. Kaviany, Principles of Heat Transfer in Porous Media, Springer, New York, NY, USA, 2nd edition, 1995.

[6] V. E. Arkhincheev, "Anomalous diffusion and charge relaxation on comb model: exact solutions," Physica A, vol. 280, no. 3, pp. 304-314, 2000.

[7] D. A. Nield and A. Bejan, Convection in Porous Media, Springer, New York, NY, USA, 3rd edition, 2006.

[8] A. S. Chaves, "A fractional diffusion equation to describe Lévy flights," Physics Letters A, vol. 239, no. 1-2, pp. 13-16, 1998.

[9] S. Jespersen, R. Metzler, and H. C. Fogedby, "Lévy flights in external force fields: Langevin and fractional Fokker-Planck equations and their solutions," Physical Review E, vol. 59, no. 3, pp. 2736-2745, 1999.

[10] V. V. Yanovsky, A. V. Chechkin, D. Schertzer, and A. V. Tur, "Lévy anomalous diffusion and fractional Fokker-Planck equation," Physica A, vol. 282, no. 1-2, pp. 13-34, 2000.

[11] D. A. Benson, S. W. Wheatcraft, and M. M. Meerschaert, "Application of a fractional advection-dispersion equation," Water Resources Research, vol. 36, no. 6, pp. 1403-1412, 2000.

[12] D. A. Benson, S. W. Wheatcraft, and M. M. Meerschaert, "The fractional-order governing equation of Lévy motion," Water Resources Research, vol. 36, no. 6, pp. 1413-1423, 2000. 
[13] J. H. Cushman and T. R. Ginn, "Fractional advection-dispersion equation: a classical mass balance with convolution-Fickian flux," Water Resources Research, vol. 36, no. 12, pp. 3763-3766, 2000.

[14] M. M. Meerschaert and C. Tadjeran, "Finite difference approximations for fractional advection-dispersion flow equations," Journal of Computational and Applied Mathematics, vol. 172, no. 1, pp. 65-77, 2004.

[15] F. Liu, V. Anh, and I. Turner, "Numerical solution of the space fractional Fokker-Planck equation," Journal of Computational and Applied Mathematics, vol. 166, no. 1, pp. 209-219, 2004.

[16] Y. Zhang, D. A. Benson, M. M. Meerschaert, and H.-P. Scheffler, "On using random walks to solve the space-fractional advection-dispersion equations," Journal of Statistical Physics, vol. 123, no. 1, pp. 89-110, 2006.

[17] Q. Liu, F. Liu, I. Turner, and V. Anh, "Approximation of the Lévy-Feller advection-dispersion process by random walk and finite difference method," Journal of Computational Physics, vol. 222, no. 1, pp. 57-70, 2007.

[18] Y. Zhang, D. A. Benson, M. M. Meerschaert, and E. M. LaBolle, "Space-fractional advection-dispersion equations with variable parameters: diverse formulas, numerical solutions, and application to the Macrodispersion Experiment site data," Water Resources Research, vol. 43, no. 5, Article ID W05439, 2007.

[19] R. Schumer, M. M. Meerschaert, and B. Baeumer, "Fractional advection-dispersion equations for modeling transport at the Earth surface," Journal of Geophysical Research, vol. 114, no. 4, Article ID F00A07, 2009.

[20] G. Jumarie, "A Fokker-Planck equation of fractional order with respect to time," Journal of Mathematical Physics, vol. 33, no. 10, pp. 3536-3542, 1992.

[21] R. Metzler, E. Barkai, and J. Klafter, "Anomalous diffusion and relaxation close to thermal equilibrium: a fractional FokkerPlanck equation approach," Physical Review Letters, vol. 82, no. 18, pp. 3563-3567, 1999.

[22] E. Barkai, R. Metzler, and J. Klafter, "From continuous time random walks to the fractional Fokker-Planck equation," Physical Review E, vol. 61, no. 1, pp. 132-138, 2000.

[23] E. Barkai, "Fractional Fokker-Planck equation, solution, and application," Physical Review E, vol. 63, no. 4, Article ID 046118, 17 pages, 2001.

[24] F. Liu, V. Anh, I. Turner, and P. Zhuang, "Time fractional advection-dispersion equation," Journal of Applied Mathematics and Computing, vol. 13, no. 1-2, pp. 233-245, 2003.

[25] F. Huang and F. Liu, "The time fractional diffusion equation and the advection-dispersion equation," ANZIAM Journal, vol. 46, no. 3, pp. 317-330, 2005.

[26] S. Momani, "An algorithm for solving the fractional convectiondiffusion equation with nonlinear source term," Communications in Nonlinear Science and Numerical Simulation, vol. 12, no. 7, pp. 1283-1290, 2007.

[27] S. Momani and A. Yıldırım, "Analytical approximate solutions of the fractional convection-diffusion equation with nonlinear source term by He's homotopy perturbation method," International Journal of Computer Mathematics, vol. 87, no. 5, pp. 10571065, 2010.

[28] G. H. Zheng and T. Wei, "Spectral regularization method for a Cauchy problem of the time fractional advection-dispersion equation," Journal of Computational and Applied Mathematics, vol. 233, no. 10, pp. 2631-2640, 2010.
[29] I. Karatay and S. R. Bayramoglu, "An efficient scheme for time fractional advection dispersion equations," Applied Mathematics Sciences, vol. 6, no. 98, pp. 4869-4878, 2012.

[30] H. Huang and X. Cao, "Numerical method for two dimensional fractional reaction subdiffusion equation," European Physical Journal, vol. 222, no. 8, pp. 1961-1973, 2013.

[31] M. Merdan, "Analytical approximate solutions of fractional convection-diffusion equation with modified RiemannLiouville derivative by means of fractional variational iteration method," Iranian Journal of Science \& Technology, no. 1, pp. 83-92, 2013.

[32] G. M. Zaslavsky, M. Edelman, and B. A. Niyazov, "Selfsimilarity, renormalization, and phase space nonuniformity of Hamiltonian chaotic dynamics," Chaos, vol. 7, no. 1, pp. 159-181, 1997.

[33] A. I. Saichev and G. M. Zaslavsky, "Fractional kinetic equations: solutions and applications," Chaos, vol. 7, no. 4, pp. 753-764, 1997.

[34] K. M. Kolwankar and A. D. Gangal, "Local fractional FokkerPlanck equation,” Physical Review Letters, vol. 80, no. 2, pp. 214217, 1998.

[35] D. Kusnezov, A. Bulgac, and G. D. Dang, "Quantum Lévy processes and fractional kinetics," Physical Review Letters, vol. 82, no. 6, pp. 1136-1139, 1999.

[36] G. M. Zaslavsky, Hamiltonian Chaos and Fractional Dynamics, Oxford University Press, New York, NY, USA, 2005.

[37] F. Liu, P. Zhuang, V. Anh, I. Turner, and K. Burrage, "Stability and convergence of the difference methods for the space-time fractional advection-diffusion equation," Applied Mathematics and Computation, vol. 191, no. 1, pp. 12-20, 2007.

[38] A. Yıldırım and H. Koçak, "Homotopy perturbation method for solving the space-time fractional advection-dispersion equation," Advances in Water Resources, vol. 32, no. 12, pp. 1711-1716, 2009.

[39] E. A. Abdel-Rehim, "Explicit approximation solutions and proof of convergence of space-time fractional advection dispersion equations," Applied Mathematics, vol. 4, no. 10, pp. 14271440, 2013.

[40] R. Gorenflo and F. Mainardi, "Fractional calculus: integral and differential equations of fractional order," in Fractals and Fractional Calculus in Continuum Mechanics, pp. 223-276, Springer, Wien, Austria, 1997.

[41] I. Podlubny, Fractional Differential Equations, Academic Press, New York, NY, USA, 1999.

[42] A. A. Kilbas, H. M. Srivastava, and J. J. Trujillo, Theory and Applications of Fractional Differential Equations, Elsevier, Amsterdam, The Netherlands, 2006.

[43] A. P. Prudnikov, Y. A. Brychkov, and O. I. Marichev, Integrals and Series. Elementary Functions, Nauka, Moscow, Russia, 1981 (Russian).

[44] Y. Z. Povstenko, "Fractional heat conduction equation and associated thermal stress," Journal of Thermal Stresses, vol. 28, no. 1, pp. 83-102, 2005.

[45] Y. Z. Povstenko, "Two-dimensional axisymmetric stresses exerted by instantaneous pulses and sources of diffusion in an infinite space in a case of time-fractional diffusion equation," International Journal of Solids and Structures, vol. 44, no. 7-8, pp. 2324-2348, 2007.

[46] R. Gorenflo, J. Loutchko, and Y. Luchko, "Computation of the Mittag-Leffler function and its derivatives," Fractional Calculus and Applied Analysis, vol. 5, no. 4, pp. 491-518, 2002. 
[47] Y. Z. Povstenko, "Stresses exerted by a source of diffusion in a case of a non-parabolic diffusion equation," International Journal of Engineering Science, vol. 43, no. 11-12, pp. 977-991, 2005.

[48] Y. Povstenko, "Signaling problem for time-fractional diffusionwave equation in a half-plane," Fractional Calculus and Applied Analysis, vol. 11, no. 3, pp. 329-352, 2008.

[49] W. R. Schneider, "Fractional diffusion," in Dynamics and Stochastic Processes, vol. 355 of Lecture Notes in Physics, pp. 276286, Springer, Berlin, Germany, 1990. 


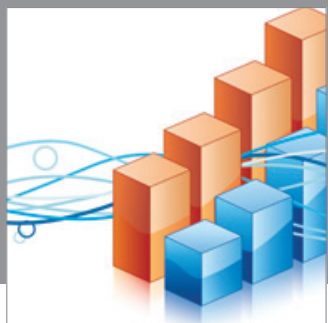

Advances in

Operations Research

mansans

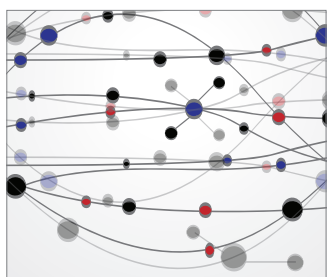

The Scientific World Journal
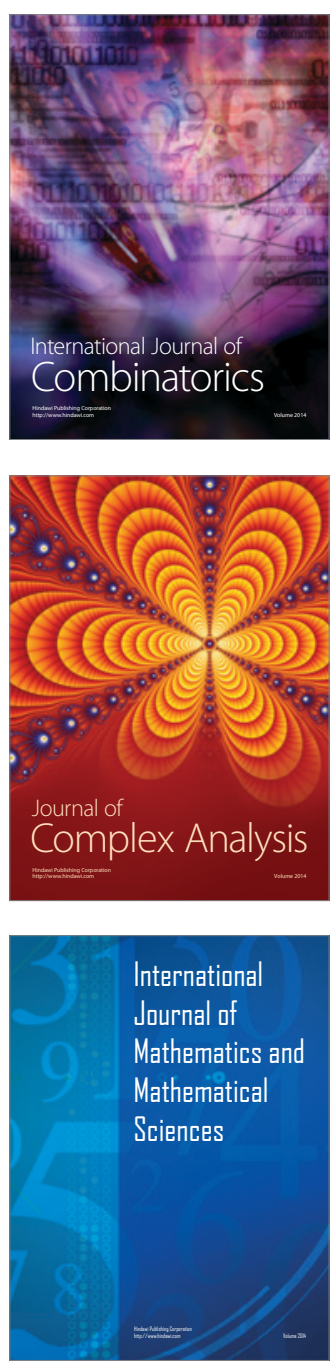
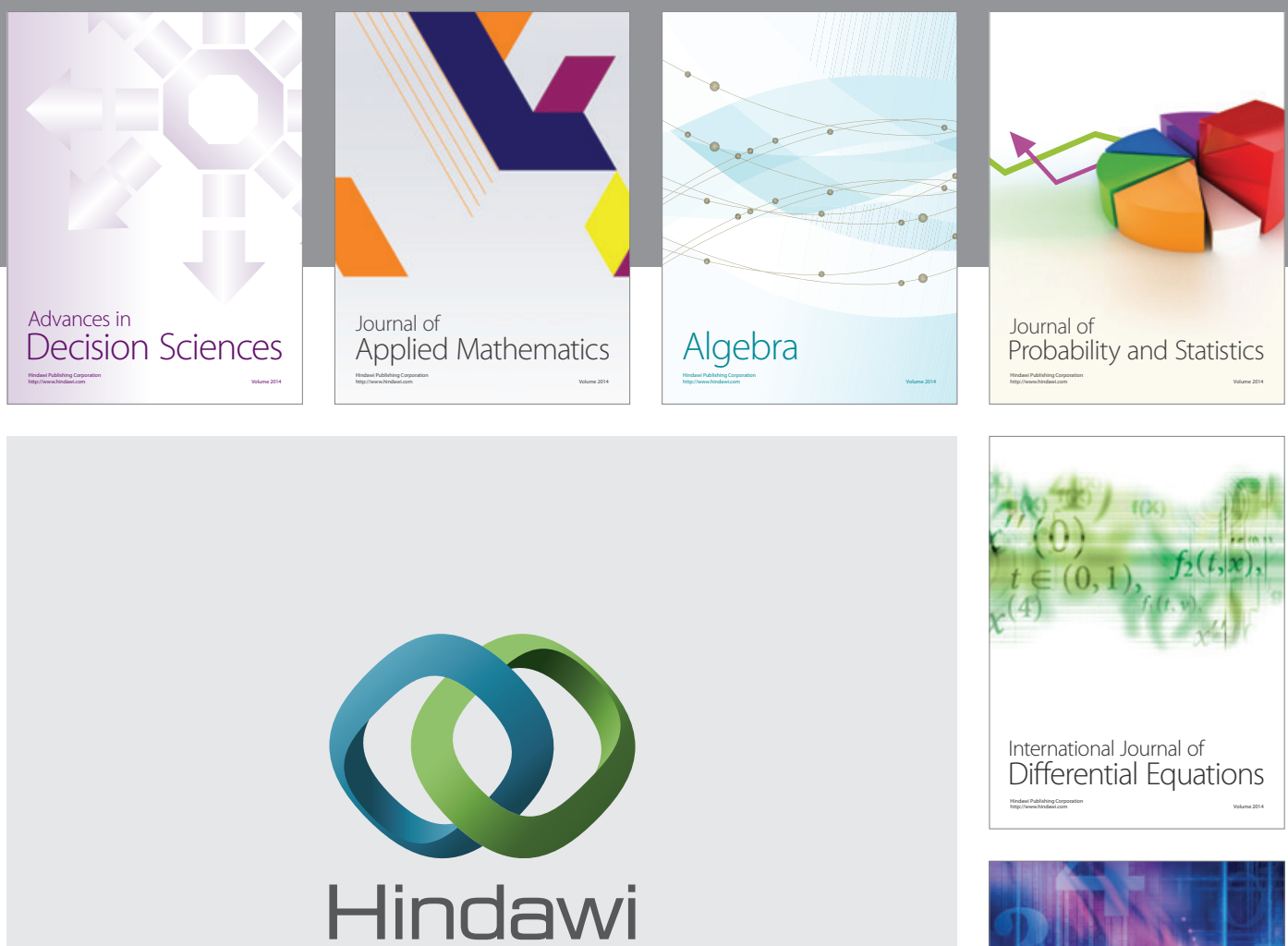

Submit your manuscripts at http://www.hindawi.com
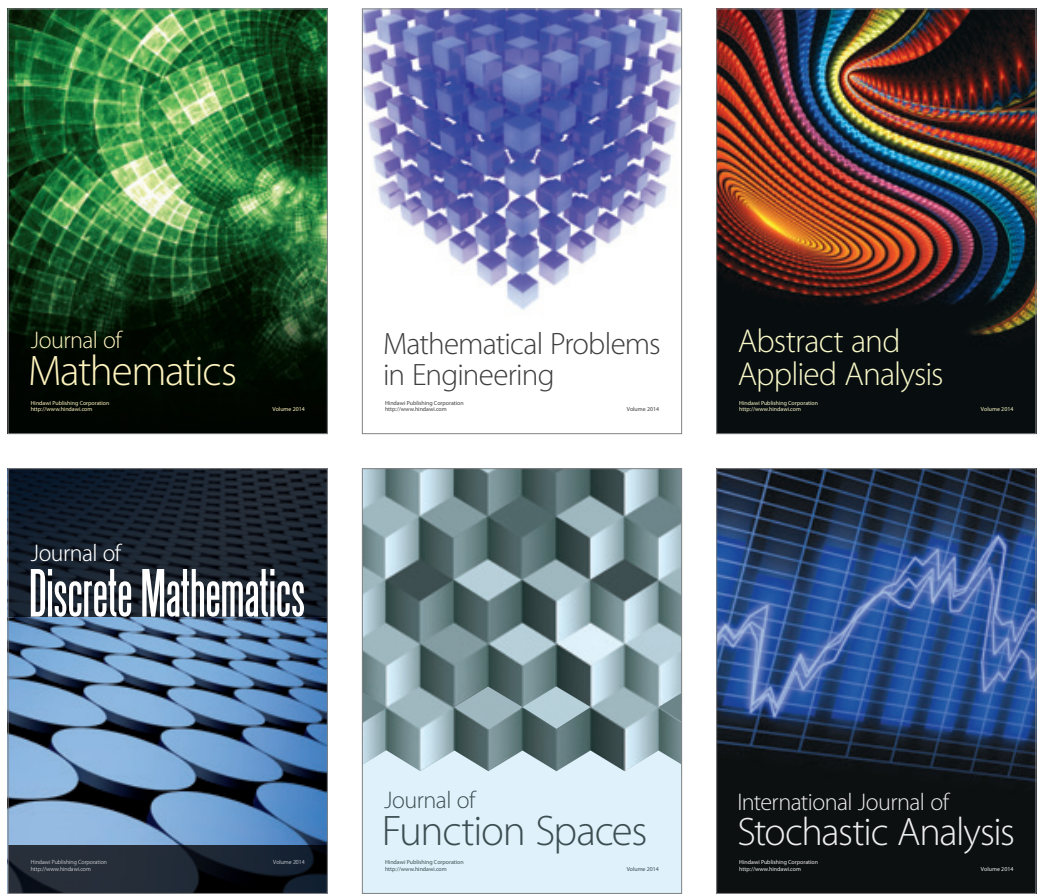

Journal of

Function Spaces

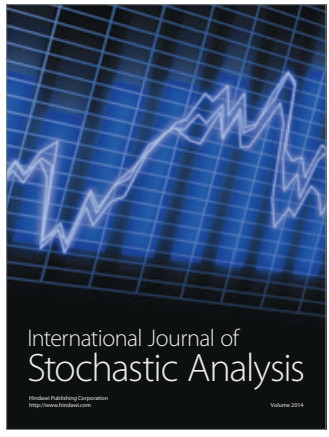

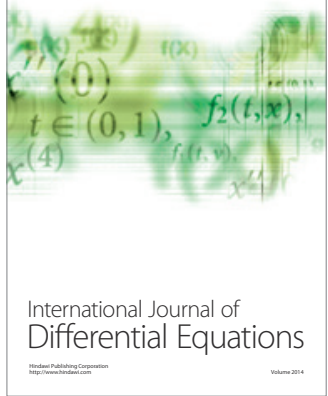
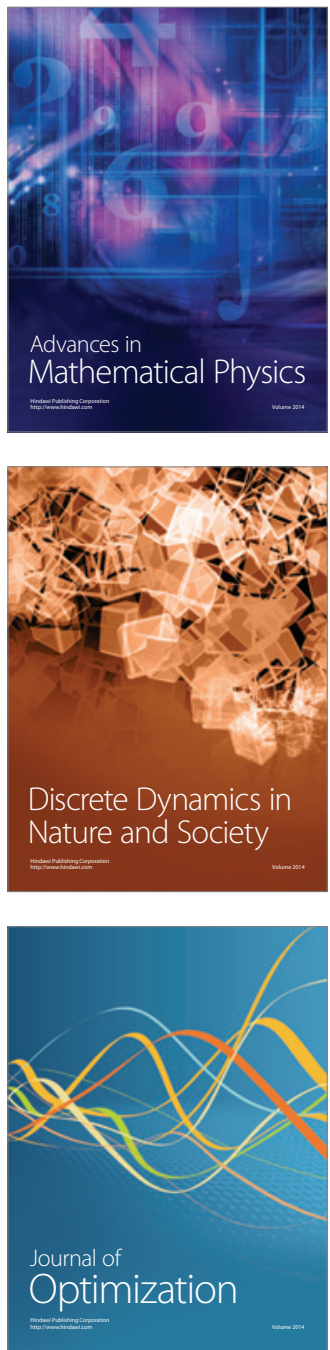